BROOKHAVEN NATIONAL LABORATORY

Assoclated Untversities, Inc. Upton, New York

ACCELERATOR DEPARTMENT

Informal Report

\title{
PERTURBATION METHOD FOR COS $\theta$ MAGNET WITH NONLINEAR IRON
}

H. Hahn

March 3, 1972

\section{NOTICE}

This report was prepared as an account of work sponsored by the United States Government. Neither the United States nor the United States Atomic Energy Commistion, nor any of their employees, nor any of their contractors, subcontractors, or their employees. makes any warranty, express or implied, or assumes any legal liability or responsibility for the accuracy, completeness or usefulness of any information, apparatus, product or process disclosed, or represents that its use would not infringe privately owned rights.

\section{$\mathrm{NOT} I \mathrm{CE}$}

This report was prepared as an account of work sponsored by the United States Government. Neither the United States nor the United States Atomic Energy Commission, nor any of their employees, nor any of their contractors, subcontractors, or their employees, makes any warranty, express or implied, or assumes any legal liability or responsibility for the accuracy, completeness or usefulness of any information, apparatus, product or process disclosed, or represents that its use would not infringe privately owned rights. 
Accelerator Department BROOKHAVEN NAT IONAL LABORATORY Asscciated Universities, Inc. Upton, New York

\title{
INTERSECTING STORAGE ACCELERATOR NOTES
}

PERTURBATION METHOD FOR $\cos \theta$ MAGNET WITH NONLINEAR IRON

\author{
H. Hahn \\ March 3, 1972
}

\section{Introduction}

The study presented here was initiated by the fact that there remains some disagreement between computed results from mesh-iteration programs 1 and the experimental results for the superconducting dipole magnet models. ${ }^{2}$ Although unlikely, the possibility of insufficient accuracy of the computer programs due to the mesh size should not completely be ruled out. It is, therefore, indicated to search for semi-analytical methods which are able to take into account the nonlinearity of the iron shield. Suggestions of this type were made by Halbach ${ }^{3}$ and Doinikov. ${ }^{4}$

Since the field-current relationship of a $\cos \theta$ magnet deviates even at $40 \mathrm{kG}$ only by a few percent from a linear law and since the induced higher harmonics are less than $1 \%$, we expect that the simple first-order perturbation method presented here should be adequate to describe the nonlinearities of the iron. The final judgement on its validity, however, must be tested through comiarison with experimental results.

1. G. Parzen and K. Jellett, Particle Accelerators 2, 169 (1971).

2. G. Parzen, CRISP 72-8.

3. K. Halbach, Nuc1. Instr. Methods 78, 185 (1970).

4. N.I. Doinikov, Sov. Phys. Tech. Phys. 15, 2030 (1971). 
The simplicity of the perturbation method suggested stems to a large extent from the simple geometry of a rotational symmetric iron shield (Fig. 1). The extension to magnets of different types, e.g. AGS magnet, picture-frame magnet, etc., is not straightforward. In this respect, the present method is inferior to mesh-type programs, which in principle can treat any geometry.

We limit our considerations to two-dimensional magnetic fields, $\vec{B}=\vec{u}_{r^{B}}{ }_{r}+\vec{u}_{\theta^{B}}$. As usual, we search for a solution for the vector potential $\vec{A}=\vec{u}_{z} A$ rather than the field directly. The field is then obtained from

$$
\vec{B}=\operatorname{cur1} \vec{A}
$$

which reads in circular-cylinder coordinates

$$
\begin{aligned}
& B_{r}=\frac{1}{r} \frac{\partial A}{\partial \theta} \\
& B_{\theta}=-\frac{\partial A}{\partial r} .
\end{aligned}
$$

The vector potential $A$ is the solution of the differential equation (DE) 5 (natural units are used throughout)

$$
\operatorname{div} \gamma \overrightarrow{\operatorname{grad}} A=-j_{\mathrm{ex}}
$$

with $j_{\text {ex }}$ being the conduction current density and $\gamma=1 / \mu$ the inverse of the permeability. $\gamma$ is assumed to be a function of the absolute value of the magnetic field, $\gamma=\gamma(B)$, but otherwise isotropic.

By using the vector identity

$$
\operatorname{div}(s \vec{v})=s \operatorname{div} \vec{v}+\vec{v} \cdot \operatorname{grad} s
$$

one can transform the DE (3) into $(\gamma=1$ at the coil location)

$$
\operatorname{div}(\operatorname{grad} A)=\Delta A=-j_{e x}-Y^{-1} \overrightarrow{\operatorname{grad}} Y \cdot \overrightarrow{\operatorname{grad} A} .
$$

The nonlinear DE (4) can be approximated by a linear Poisson equation, if the right-hand side of (4) is evaluated for a trial function which is not too different from the correct solution. Since the nonlinearities represent a small

5. L.D. Landau and E.M. Lifshitz, Electrodynamics of Cont inuous Media, p. 121 
perturbation to the 1 inear case $Y=0$, a good approximation is expected by using the linear solution as trial function. Discontinuities in $y$ modify Eq. (4) slightly, but not the principle of our method.

\section{Air-Core Magnet}

In this section a method for the analysis of the magnetic field due to an extraneous current distribution is elaborated, which matches the geometry of air$\operatorname{core} \cos \theta$ magnets (Fig. 1). The current density $j_{e x}=j_{e x}(r, \theta)$ is 1 imited to the region $r_{i}<r<r_{0}$ and exhibits in the absence of fabrication errors the symmetry properties

$$
j_{e x}(r, \theta)=j_{e x}(r,-\theta)
$$

and

$$
j_{\mathrm{ex}}(r, \theta)=-j_{\mathrm{ex}}(r, \theta+\pi)
$$

Considerable simplifications are achieved by representing the current density through the Fourier series which has the general form

$$
j_{e x}=\sum_{n=1}^{\infty} J_{n}(r) \cos n \theta \text { for } r_{i}<r<r_{0}
$$

with $n$ restricted to odd integers. (We will use the convention that $\sum_{0}$ expresses a sum over odd $n$ and $\sum_{e}$ a sum over even $\left.n_{.}\right)$For certain geometries, the Fourier analysis of the current distribution can be done analytically, for others numerically only. In any case, the radially dependent functions $J_{n}$ are obtained by simple integration according to

$$
J_{n}(r)=\frac{1}{\pi} \int_{0}^{2 \pi} j_{e x}(r, \theta) \cos n \theta d \theta .
$$

The vector potential satisfies the DE (4) which in the absence of the iron shield is simply

$$
\Delta A^{e x}=-j_{e x} .
$$

In view of the Fourier representation of the forcing term it is advantageous to make the ansatz for the vector potential

$$
A^{e x}=\sum_{\rho} A_{n}^{e x}(r) \cos n \theta \text {. }
$$


The functions $A_{n}^{e x}(r)$ must then each satisfy the ordinary $D E$

$$
\frac{1}{r} \frac{d}{d r}\left(r \frac{d A_{n}^{e x}}{d r}\right)-\frac{n^{2}}{r^{2}} A_{n}^{e x}=\left\{\begin{array}{cl}
-J_{n}(r) & \text { for } r_{i}<r<r_{0} \\
0 & \text { for } r<r_{i} ; r>r_{0}
\end{array}\right.
$$

together with the boundary conditions that $A_{n}^{e x}$ and $d_{A}{ }_{n}^{e x} / d r$ is continuous at $\mathbf{r}_{\mathbf{i}}$ and $\mathbf{r}_{\mathbf{0}}$.

After simple manipulations one obtains

- for $r<r_{i}$

$$
\begin{aligned}
& A_{n}^{\text {ex }}=\frac{1}{2 n}\left(\frac{r}{r_{i}}\right)^{n} s_{n}^{\text {tex }} r_{i} \\
& \frac{d A_{n}^{e x}}{d r}=\frac{1}{2}\left(\frac{r}{r_{i}}\right)^{n-1} s_{n}^{\text {tex }}
\end{aligned}
$$

- for $\mathbf{r}>\mathbf{r}_{0}$

$$
\begin{aligned}
& A_{n}^{e x}=\frac{1}{2 n}\left(\frac{r_{i}}{r}\right)^{n} s_{n}^{e x} r_{i} \\
& \frac{d_{n}^{e x}}{d r}=-\frac{1}{2}\left(\frac{r_{i}}{r}\right)^{n+1} s_{n}^{e x}
\end{aligned}
$$

- and for $r_{i}<r<r_{0}$

$$
\begin{aligned}
& A_{n}^{\text {ex }}=\frac{1}{2 n}\left(\frac{r}{r_{i}}\right)^{n} r_{i}\left[s_{n}^{\text {tex }}-\sigma_{n}^{\text {tex }}(r)\right]+\frac{1}{2 n}\left(\frac{r_{i}}{r}\right)^{n} r_{i} \sigma_{n}^{e x}(r) \\
& \frac{d A_{n}^{e x}}{d r}=\frac{1}{2}\left(\frac{r}{r_{i}}\right)^{n-1}\left[s_{n}^{\text {tex }}-\sigma_{n}^{+e x}(r)\right]-\frac{1}{2}\left(\frac{r_{i}}{r}\right)^{n+1} \sigma_{n}^{e x}(r)
\end{aligned}
$$

in which we defined

$$
\begin{aligned}
& \sigma_{n}^{+e x}(r)=r_{i}^{n-1} \int_{r_{i}}^{r} J_{n}(r) r^{-(n-1)} d r \\
& \sigma_{n}^{e x}(r)=r_{i}^{-(n+1)} \int_{r_{i}}^{r} J_{n}(r) r^{n+1} d r
\end{aligned}
$$


and

$$
\begin{aligned}
& s_{n}^{t e x}=\sigma_{n}^{t e x}\left(r_{0}\right) \\
& s_{n}^{e x}=\sigma_{n}^{e x}\left(r_{0}\right) .
\end{aligned}
$$

Obviously,

$$
\sigma_{n}^{+e x}\left(r_{i}\right)=\sigma_{n}^{e x}\left(r_{i}\right)=0
$$

For the subsequent development of this paper only the knowledge of the coefficients $S_{n}$ is required, and it is immaterial how they were obtained. It is, for example, possible to use the results of Morgan's ${ }^{6}$ MAGFLD program for the air-core case and proceed as out lined in the sequel. It would, however, appear to us that eventually the above approach is more economical and represents the geometry of a $\cos \theta$ magnet more directly.

\section{Presence of Constant Permeability Iron Shield}

In the presence of a constant permeability iron shield the solution for the vector potential is usually found from $\mathrm{DE}$ (7) together with the boundary condi$t$ ion that $A$ and $\gamma d A / d r$ are continuous at the iron-air interfaces. An alternate approach, more suited to the subsequent development, consists in introducing induced magnetization currents at the interface. The vector potential is now determined by the $\mathrm{DE}$

$$
\Delta A=-j_{e x}-G^{R i} \delta\left(R_{i}\right)-G^{R o} \delta\left(R_{0}\right)
$$

where $\delta(R)$ is the delta function with the property

$$
\int \delta(R) d r=1
$$

Consistency requires that the line currents $G$ satisfy the side conditions

$$
\begin{aligned}
& G^{R i}=\left[(\gamma-1) \frac{\partial A}{\partial r}\right]_{R_{i}+\varepsilon} \\
& G^{R o}=\left[(1-\gamma) \frac{\partial A}{\partial r}\right]_{R_{0}-\varepsilon}
\end{aligned}
$$

6. G.H. Morgan, Brookhaven National Laboratory, Accelerator Dept. Reports AADD-145 (1968) and AADD-145B (1970). 
which follow from (4) for discontinuities in $\gamma$, but otherwise $\gamma=$ constant or unity.

Separating the effects due to the extraneols currents and the induced magnetization currents one can write $A=A A^{e x}+A^{F e}$. Subtracting DE (7) from (12) leads to the DE for $A=\sum_{n} A_{n}^{F e}(r) \cos n \theta$

$$
\Delta A^{F e}=-G^{R i} \delta\left(R_{i}\right)-G^{R o} \delta\left(R_{0}\right)
$$

with the solution

- for $\mathbf{r}<\mathbf{R}_{\mathbf{i}}$

$$
\begin{aligned}
& A_{n}^{F e}=\frac{1}{2 n}\left\{\left(\frac{r}{R_{i}}\right)^{n} G_{n}^{R i} R_{i}+\left(\frac{r}{R_{0}}\right)^{n} G_{n}^{R o} R_{0}\right\} \\
& \frac{d A_{n}^{F e}}{d r}=\frac{1}{2}\left\{\left(\frac{r}{R_{i}}\right)^{n-1} G_{n}^{R i}+\left(\frac{r}{R_{0}}\right)^{n-1} G_{n}^{R o}\right\}
\end{aligned}
$$

- for $R_{i}<r<R_{0}$

$$
\begin{aligned}
& A_{n}^{F e}=\frac{1}{2 n}\left\{\left(\frac{R_{i}}{r}\right)^{n} G_{n}^{R i} R_{i}+\left(\frac{r}{R_{0}}\right)^{n} G_{n}^{R o} R_{0}\right\} \\
& \frac{d A_{n}^{F e}}{d r}=\frac{1}{2}\left\{-\left(\frac{R_{i}}{r}\right)^{n+1} G_{n}^{R i}+\left(\frac{r}{R_{0}}\right)^{n-1} G_{n}^{R o}\right\}
\end{aligned}
$$

- and for $r>R_{0}$

$$
\begin{aligned}
& A_{n}^{F e}=\frac{1}{2 n}\left\{\left(\frac{R_{i}}{r}\right)^{n} G_{n}^{R i} R_{i}+\left(\frac{R_{0}}{r}\right)^{n} G_{n}^{R o} R_{0}\right\} \\
& \frac{d A_{n}^{F e}}{d r}=-\frac{1}{2}\left\{\left(\frac{R_{i}}{r}\right)^{n+1} G_{n}^{R i}+\left(\frac{R_{0}}{r}\right)^{n+1} G_{n}^{R o}\right\} .
\end{aligned}
$$

The magnitude of the induced magnetization currents is given by (13), from which follows the coupled set of linear equations in the $G_{n}^{R i}$ and $G_{n}^{R o}$ 
$(1+\gamma) G_{n}^{R i}+(1-\gamma)\left(\frac{R_{1}}{R_{0}}\right)^{n-1} G_{n}^{R o}=(1-\gamma) s_{n}^{\operatorname{ex}}\left(\frac{r_{i}}{R_{i}}\right)^{n+1}$

$$
(1-\gamma)\left(\frac{R_{i}}{R_{0}}\right)^{n+1} G_{n}^{R i}+(1+\gamma) G_{n}^{R o}=-(1-\gamma) s_{n}^{\text {ex }}\left(\frac{r_{i}}{R_{0}}\right)^{n+1} \text {. }
$$

Separation of the coefficients is possible, leading to

$$
\begin{aligned}
& G_{n}^{R i}=s_{n}^{\operatorname{ex}}\left(\frac{r_{i}}{R_{i}}\right)^{n+1} \frac{\left\{\Gamma+\Gamma^{2}\left(R_{i} / R_{0}\right)^{2 n}\right\}}{\left\{1-\Gamma^{2}\left(R_{i} / R_{0}\right)^{2 n}\right\}} \\
& G_{n}^{R o}=-s_{n}^{e x}\left(\frac{r_{i}}{R_{0}}\right)^{n+1} \frac{\left\{\Gamma+\Gamma^{2}\right\}}{\left\{1-\Gamma^{2}\left(R_{i} / R_{0}\right)^{2 n}\right\}} .
\end{aligned}
$$

The presence of the iron shield increases the desired field inside the current coils. According to (15) the increase is given by $\left(r<r_{i}\right)$

$$
\begin{aligned}
& A_{n}^{F e}=\frac{1}{2 n}\left(\frac{r}{r_{i}}\right)^{n}\left(\frac{r_{i}}{R_{i}}\right)^{2 n} s_{n}^{e x} r_{i} \Gamma \frac{1-\left(R_{i} / R_{0}\right)^{2 n}}{1-\Gamma^{2}\left(R_{i} / R_{0}\right)^{2 n}} \\
& \frac{d A_{n}^{F e}}{d r}=\frac{1}{2}\left(\frac{r}{r_{i}}\right)^{n-1}\left(\frac{r_{i}}{R_{i}}\right)^{2 n} s_{n}^{\operatorname{ex}} \Gamma \frac{1-\left(R_{i} / R_{0}\right)^{2 n}}{1-\Gamma^{2}\left(R_{i} / R_{0}\right)^{2 n}} .
\end{aligned}
$$

Obviously the maximum gain which can be achieved is for infinite permeability iron, i.e., for $\gamma \rightarrow 0$ and $\Gamma \rightarrow 1$, in which case one finds

$$
\begin{aligned}
& A_{n}^{F e^{\infty}}=\frac{1}{2 n}\left(\frac{r}{r_{i}}\right)^{n}\left(\frac{r_{i}}{R_{i}}\right)^{2 n} s_{n}^{e x} r_{i} \\
& \frac{d A_{n}^{F e \infty}}{d r}=\frac{1}{2}\left(\frac{r}{r_{i}}\right)^{n-1}\left(\frac{r_{i}}{R_{i}}\right)^{2 n} s_{n}^{e x} .
\end{aligned}
$$

It is worth noting that for infinite permeability the outer radius does not enter into the expressions which one would expect from intuition. The flux density in the iron is, however, dependent on $R_{0}$. 
Starting from the infinite permeability case and increasing the Inverse permeability from $0: 0$ y reduces the field by $\left(r<r_{1}\right)$

$$
\begin{aligned}
& \delta A_{n}=\frac{1}{2 n}\left(\frac{r}{r_{i}}\right)^{n}\left(\frac{r_{i}}{R_{i}}\right)^{2 n} s_{n}^{e x} r_{i}(\Gamma-1) \frac{1+\Gamma\left(R_{i} / R_{o}\right)^{2 n}}{1-\Gamma^{2}\left(R_{i} / R_{o}\right)^{2 n}} \\
& \frac{d \delta A_{n}}{d r}=\frac{1}{2}\left(\frac{r}{r_{i}}\right)^{n-1}\left(\frac{r_{i}}{R_{i}}\right)^{2 n} s_{n}^{e x}(\Gamma-1) \frac{1+\Gamma\left(R_{i} / R_{o}\right)^{2 n}}{1-\Gamma^{2}\left(R_{i} / R_{o}\right)^{2 n}}
\end{aligned}
$$

The expressions for the total field within the constant permeability iron shield $\left(R_{i}<r<R_{0}\right)$ will. be required in the subsequent analysis. After some manipulations we find

$$
\begin{aligned}
& A_{n}=\frac{1}{2 n} s_{n}^{e x} r_{i} \frac{(1+\Gamma)\left\{\left(\frac{r_{i}}{r}\right)^{n}-\Gamma\left(\frac{r}{R_{0}}\right)^{n}\left(\frac{r_{i}}{R_{0}}\right)^{n}\right\}}{1-\Gamma^{2}\left(\frac{R_{i}}{R_{0}}\right)^{2 n}} \\
& \frac{d A_{n}}{d r}=-\frac{1}{2} s_{n}^{e x} \frac{(1+\Gamma)\left\{\left(\frac{r_{i}}{r}\right)^{n+1}+\Gamma\left(\frac{r}{R_{0}}\right)^{n}\left(\frac{r_{i}}{R_{0}}\right)^{n}\right\}}{1-\Gamma^{2}\left(\frac{R_{i}}{R_{0}}\right)^{2 n}} .
\end{aligned}
$$

Again we find for $\gamma \rightarrow 1$ the solution in the absence of the iron shield. The case of infinite permeability deserves special attention, and we obtain explicitly

$$
\begin{aligned}
& A^{\infty}=\frac{s_{n}^{e x} r_{i}}{n\left[1-\left(\frac{R_{i}}{R_{0}}\right)^{2 n}\right]}\left\{\left(\frac{r_{i}}{r}\right)^{n}-\left(\frac{r}{R_{0}}\right)^{n}\left(\frac{r_{i}}{R_{0}}\right)^{n}\right\} \\
& \frac{d_{A}^{\infty}}{d r}=\frac{-s_{n}^{e x}}{1-\left(\frac{R_{i}}{R_{0}}\right)^{2 n}}\left\{\left(\frac{r_{i}}{r}\right)^{n+1}+\left(\frac{r}{R_{0}}\right)^{n-1}\left(\frac{r_{i}}{R_{0}}\right)^{n+1}\right\} .
\end{aligned}
$$

It should be noted that $A^{\infty}=0$ but $d A^{\infty} / d r \neq 0$ for $r \cdots R_{0}$, which means that, indeed, the magnetic field is parallel to the surface and vanishes outside. 
The leakage field outside the fron shield is easily measured and a particularly sensitive indication as to the accuracy of the theoretical solution. We find for the constant permeability case outside the iron ( $r>R_{0}$ )

$$
\begin{aligned}
& A_{n}=\frac{1}{2 n} \frac{1-\Gamma^{2}}{1-\Gamma^{2}\left(R_{i} / R_{0}\right)^{2 n}}\left(\frac{r_{i}}{r}\right)^{n} s_{n}^{e x} r_{i} \\
& \frac{d A_{n}}{d r}=-\frac{1}{2} \frac{1-\Gamma^{2}}{1-\Gamma^{2}\left(R_{i} / R_{0}\right)^{2 n}}\left(\frac{r_{i}}{r}\right)^{n+1} s_{n}^{e x} .
\end{aligned}
$$

If $\gamma \rightarrow 1, \Gamma \rightarrow 0$ and one obtains the solution characteristic for the absence of an iron shield. If $Y \rightarrow 0, \Gamma \rightarrow 1$ and one obtains a leakage field $A \rightarrow 0$ as expected for the infinite permeability case.

\section{Perturbation Due to Nonlinear Iron}

The exact DE for the vector potential in the presence of a nonlinear iron shield follows from (4) and (12) as

$$
\begin{aligned}
\Delta A= & -j_{e x}-\gamma^{-1} \operatorname{grad} \gamma \cdot \operatorname{grad} A \\
& -\left[(\gamma-1) \frac{\partial A}{\partial r}\right]_{R_{i}+\epsilon} \delta\left(R_{i}\right)-\left[(1-\gamma) \frac{\partial A}{\partial r}\right]_{R_{0}-\epsilon} \delta\left(R_{0}\right) .
\end{aligned}
$$

The inverse permeability is now a function of the absolute value of the local magnetic field $B$ or, in view of (2), a function of the absolute value of the gradient of the vector potential, $|\overrightarrow{g r a d} A|$, since

$$
B=\sqrt{\left(\frac{\partial A}{\partial r}\right)^{2}+\left(\frac{1}{r} \frac{\partial A}{\partial \theta}\right)^{2}} .
$$

It was pointed out in the introduction that the nonlinear solution differs not too much from a linear solution, as a matter of fact, from the infinite permeability case. It is, therefore, natural to consider the finite permeability as well as the nonlinear case as a perturbation of the infinite permeability case,

$$
A=A^{\infty}+\delta A
$$

with $A^{\infty}$ in the iron region given by (22). 
In order to obtain the $D E$ for the perturbation $\delta A$ we subtract

$$
\Delta A^{\infty}=-j_{\text {ex }}-\left[-\frac{\partial A^{\infty}}{\partial r}\right]_{R_{i}+\epsilon} \delta\left(R_{i}\right)-\left[\frac{\partial A^{\infty}}{\partial r}\right]_{R_{0}-\epsilon} \delta\left(R_{0}\right)
$$

from $D E(24)$ and find the rigorous $D E$

$$
\begin{aligned}
\Delta \delta A= & -\gamma^{-1} \operatorname{grad} \gamma \cdot \overrightarrow{g r a d} A \\
& -\left[-\frac{\partial \delta A}{\partial r}+\gamma \frac{\partial A}{\partial r}\right]_{R_{i}+\epsilon} \delta\left(R_{i}\right)-\left[\frac{\partial \delta A}{\partial r}-\gamma \frac{\partial A}{\partial r}\right]_{R_{0}-\epsilon} \delta\left(R_{0}\right) .
\end{aligned}
$$

It is now possible to simplify DE (26) considerably by replacing a known approximate solution $A^{\text {trial }}$ for $A$. It is natural to start with $A^{\text {trial }} \approx A^{\infty}$, but if by some other way (iteration procedure) a better solution is known, it could be used. By making this approximation one can reduce the nonlinear DE to a linear equation resembling the Poisson equation. The approximate DE describing the nonlinear iron takes the form

$$
\Delta \delta A=-\gamma^{-1} \overrightarrow{\operatorname{grad}} \gamma \cdot \operatorname{grad} A^{\infty}-g^{R i} \delta\left(R_{i}\right)-g^{R o} \delta\left(R_{0}\right)
$$

with the constants

$$
\begin{aligned}
& \mathbf{g}^{\mathrm{Ri}}=\left[-\frac{\partial \delta A}{\partial r}+\gamma \frac{\partial A^{\infty}}{\partial r}\right]_{\mathbf{R}_{i}+\epsilon} \\
& \mathbf{g}^{\mathrm{Ro}}=\left[\frac{\partial \delta A}{\partial r}-\gamma \frac{\partial A^{\infty}}{\partial r}\right]_{\mathbf{R}_{0}-\epsilon} .
\end{aligned}
$$

In order to test the accuracy of the solution and to demonstrate the perturbation method by means of a known result we will now reconsider the case of a finite constant permeability iron shield. From $\gamma=$ const it follows that $\overrightarrow{\operatorname{grad}} \gamma=C$. With the usual ansatz $\delta A=\sum_{e} \delta A_{n} \cos n \theta$ follows the solution within the iron $\left(R_{1}<r<R_{0}\right)$

$$
\begin{aligned}
& \delta A_{n}=\frac{1}{2 n}\left\{\left(\frac{R_{i}}{r}\right)^{n} g_{n}^{R i} R_{i}+\left(\frac{r}{R_{0}}\right)^{n} g_{n}^{R o} R_{0}\right\} \\
& \frac{d \delta A_{n}}{d r}=\frac{1}{2}\left\{-\left(\frac{R_{i}}{r}\right)^{n+1} g_{n}^{R i}+\left(\frac{r}{R_{0}}\right)^{n-1} g_{n}^{R i}\right\} .
\end{aligned}
$$


Using the expression for $A^{\infty}$ from (22) we arrive at the set of 1 inear equations in $\mathrm{g}_{\mathrm{n}}^{\mathrm{i}}$ and $\mathrm{g}_{\mathrm{n}}^{\mathrm{Ro}}$

$g_{n}^{R i}=\frac{1}{2}\left\{g_{n}^{R i}-\left(\frac{R_{i}}{R_{0}}\right)^{n-1} g_{n}^{R o}\right\}-\gamma \frac{s_{n}^{e x}}{1-\left(R_{i} / R_{0}\right)^{2 n}}\left\{\left(\frac{r_{i}}{R_{i}}\right)^{n+1}+\left(\frac{R_{i}}{R_{0}}\right)^{n-1}\left(\frac{r_{i}}{R_{0}}\right)^{n+1}\right\}$

$g_{n}^{R o}=\frac{1}{2}\left\{-\left(\frac{R_{i}}{R_{0}}\right)^{n+1} g_{n}^{R i}+g_{n}^{R o}\right\}+\gamma \frac{s_{n}^{e x}}{1-\left(R_{i} / R_{0}\right)^{2 n}}\left\{2\left(\frac{r_{i}}{R_{0}}\right)^{n+1}\right\}$

with the solution

$$
\begin{aligned}
& g_{n}^{R i}=-2 y \frac{s_{n}^{e x}}{\left[1-\left(R_{i} / R_{0}\right)^{2 n}\right]^{2}}\left(\frac{r_{1}}{R_{i}}\right)^{n+1}\left\{1+3\left(\frac{R_{i}}{R_{0}}\right)^{2 n}\right\} \\
& g_{n}^{R o}=2 \gamma \frac{s_{n}^{e x}}{\left[1-\left(R_{i} / R_{0}\right)^{2 n}\right]^{2}}\left(\frac{\varepsilon_{i}}{R_{0}}\right)^{n+1}\left\{3+\left(\frac{R_{i}}{R_{0}}\right)^{2 n}\right\} .
\end{aligned}
$$

The leakage field $\left(r>R_{0}\right.$ ) for infinite permeability is zero and increases for finite $Y$ to a value given by

$$
\begin{aligned}
& \delta A_{n}=\frac{1}{2 n}\left\{\left(\frac{R_{i}}{r}\right)^{n} g_{n}^{R i} R_{i}+\left(\frac{R_{0}}{r}\right)^{n} g_{n}^{R o} R_{0}\right\} \\
& \frac{d \delta A_{n}}{d r}=-\frac{1}{2}\left\{\left(\frac{R_{i}}{r}\right)^{n+1} g_{n}^{R i}+\left(\frac{R_{0}}{r}\right)^{n+1} g_{n}^{R o}\right\} .
\end{aligned}
$$

After substitution of $g_{n}^{R i}$ and $g_{n}^{R o}$ from (30) follows

$$
\begin{aligned}
& \delta A_{n}=\frac{1}{2 n}\left(\frac{r_{i}}{r}\right)^{n} \frac{4 \gamma s_{n}^{e x} r_{i}}{1-\left(R_{i} / R_{0}\right)^{2}} \\
& \frac{d \delta A_{n}}{d r}=-\frac{1}{2}\left(\frac{r_{i}}{r}\right)^{n+1} \frac{4 \gamma s_{n}^{e x}}{1-\left(R_{i} / R_{0}\right)^{2}}
\end{aligned}
$$

which is in first order identical to the exact solution given by (23) since $1-\Gamma^{2} \approx 4 \gamma+\ldots$. In the same way, one can show that the perturbation theory gives in first order the correct result for the decrease of the field inside the coils, as expressed by Eq. (20). In view of this agreement, we expect that the accuracy of the perturbation method is adequate for all practical purposes. 
Proceeding towards the general solution of DE (27), we have to obtain the Fourier series

$$
\gamma=\gamma_{0}(r)+\sum e \gamma_{n}(r) \cos n \theta
$$

and correspondingly

$$
\begin{aligned}
\gamma^{-1} \operatorname{grad} \gamma & =\vec{u}_{r}\left\{M_{0}^{r}(r)+\sum_{n} M_{n}^{r}(r) \cos n \theta\right\} \\
& +\vec{u}_{\theta}\left\{\sum_{n} M_{n}^{\theta}(r) \sin n \theta\right\}
\end{aligned}
$$

The determination of the functions $M_{n}^{r}, M_{n}^{\theta}$ and $\gamma_{n}$ represents the bulk of the computational work. Once they are known, it is relatively easy to find the complete solution for the $\delta A_{n}=\delta A_{n}(r)$ from the ordinary $D E$

$$
\frac{1}{r} \frac{d}{d r}\left[r \frac{d \delta A_{n}}{d r}\right]-\frac{n^{2}}{r^{2}} \delta A_{n}=-g_{n}^{R i} \delta\left(R_{i}\right)-g_{n}^{R o} \delta\left(R_{o}\right)-I_{n}(r)
$$

in which $g_{n}^{R i}$ and $g_{n}^{R o}$ are constants to be determined by consistency conditions and $I_{n}(r)$ is a known function given below.

To avoid lengthy expressions and without practical consequence we now limit our discussion to "good" $\cos \theta$ magnets, that is, we assume $A^{\infty} \approx A_{1}^{\infty} \cos \theta$ in the subsequent expressions. Note, however, that $\gamma$ and $\gamma^{-1} \operatorname{grad} \gamma$ was determined for the exact $A^{\infty}$. With the above simplification we obtain

$$
\begin{aligned}
& I_{1}(r)=\frac{d A_{1}^{\infty}(r)}{d r}\left\{M_{0}^{r}(r)+\frac{1}{2} M_{2}^{r}(r)\right\}-\frac{A_{1}^{\infty}(r)}{r}\left\{\frac{1}{2} M_{2}^{\theta}(r)\right\} \\
& I_{n}(r)=\frac{d A_{1}^{\infty}(r)}{d r}\left\{\frac{1}{2} M_{n-1}^{r}(r)+\frac{1}{2} M_{n+1}^{r}(r)\right\}-\frac{A_{1}^{\infty}(r)}{r}\left\{\frac{1}{2} M_{n-1}^{\theta}(r)-\frac{1}{2} M_{n+1}^{\theta}(r)\right\} .
\end{aligned}
$$

The solution of (35) within the iron shield $\left(R_{i}<r<R_{0}\right)$ has the general form 


$$
\begin{aligned}
\delta A_{n} & =\frac{1}{2 n}\left\{\left(\frac{R_{1}}{r}\right)^{n} g_{n}^{R i} R_{i}+\left(\frac{r}{R_{0}}\right)^{n} g_{n}^{R o} R_{0}\right\} \\
& +\frac{1}{2 n}\left(\frac{r}{R_{1}}\right)^{n} R_{i}\left\{s_{n}^{\dagger} \cdot \sigma_{n}^{\dagger}(r)\right\}+\frac{1}{2 n}\left(\frac{R_{i}}{r}\right)^{n} R_{i} \sigma_{n}(r)
\end{aligned}
$$

$$
\begin{aligned}
\frac{d \delta A_{n}}{d r} & =\frac{1}{2}\left\{-\left(\frac{R_{i}}{r}\right)^{n+1} g_{n}^{R i}+\left(\frac{r}{R_{0}}\right)^{n-1} g_{n}^{R o}\right\} \\
& +\frac{1}{2}\left(\frac{r}{R_{i}}\right)^{n-1}\left\{s_{n}^{+}-\sigma_{n}^{+}(r)\right\}-\frac{1}{2}\left(\frac{R_{i}}{r}\right)^{n+1} \sigma_{n}(r)
\end{aligned}
$$

in which

$$
\begin{aligned}
& \sigma_{n}^{\dagger}(r)=R_{i}^{n-1} \int_{R_{i}}^{r} I_{n}(r) r^{-(n-1)} d r \\
& \sigma_{n}(r)=R_{i}^{-(n+1)} \int_{R_{i}}^{r} I_{n}(r) r^{n+1} d r
\end{aligned}
$$

and

$$
\begin{aligned}
& s_{n}^{+}=\sigma_{n}^{+}\left(R_{0}\right) \\
& s_{n}=\sigma_{n}\left(R_{0}\right) .
\end{aligned}
$$

Obviously $\sigma_{n}^{\dagger}\left(R_{i}\right)=\sigma_{n}\left(R_{i}\right)=0$

Internal consistency of the solution is ensured if the constants $g_{n}^{R i}$ and $g_{n}^{\text {Ro }}$ satisfy (27), from which the following coupled sets of linear equations are obtained

$$
\begin{aligned}
& g_{n}^{R i}=\frac{1}{2}\left\{g_{n}^{R i}-\left(\frac{R_{i}}{R_{0}}\right)^{n-1} g_{n}^{R o}\right\}-\frac{1}{2} s_{n}^{\dagger}+F_{n}^{R i} \\
& g_{n}^{R o}=\frac{1}{2}\left\{g_{n}^{R o}-\left(\frac{R_{1}}{R_{0}}\right)^{n+1} g_{n}^{R i}\right\}-\frac{1}{2}\left(\frac{R_{1}}{R_{0}}\right)^{n+1} s_{n}-F_{n}^{R o}
\end{aligned}
$$

in which 


$$
\begin{aligned}
& F_{1}^{R i}=\left\{\gamma_{0}\left(R_{i}\right)+\frac{1}{2} \gamma_{2}\left(R_{i}\right)\right\}\left[\frac{d A_{1}^{\infty}}{d r}\right]_{R_{1}+\epsilon} \\
& F_{n}^{R i}=\left\{\frac{1}{2} Y_{n-1}\left(R_{i}\right)+\frac{1}{2} Y_{n+1}\left(R_{i}\right)\right\}\left[\frac{d A_{1}^{\infty}}{d r}\right]_{R_{i}+\epsilon} ; \text { for } n>1 \\
& \left.F_{1}^{R o}=\left\{Y_{0}\left(R_{0}\right)+\frac{1}{2} Y_{2}\left(R_{0}\right)\right\}\left[\frac{d A_{1}^{\infty}}{d r}\right]_{R_{0}-\epsilon}\right]_{n}^{R o}=\left\{\frac{1}{2} Y_{n-1}\left(R_{0}\right)+\frac{1}{2} Y_{n+1}\left(R_{0}\right)\right\}\left[\frac{d A_{1}^{\infty}}{d r}\right]_{R_{0}-\epsilon} ; \text { for } n>1 .
\end{aligned}
$$

The values of the constants $g_{n}^{R i}$ and $g_{n}^{R o}$ can now be indicated explicitly as

$$
\begin{aligned}
& \left\{1-\left(\frac{R_{i}}{R_{0}}\right)^{2 n}\right\} g_{n}^{R i}=-s_{n}^{+}+\left(\frac{R_{1}}{R_{0}}\right)^{2 n} s_{n}+2 F_{i}^{R i}+2\left(\frac{R_{i}}{R_{0}}\right)^{n-1} F_{n}^{R o} \\
& \left\{1-\left(\frac{R_{i}}{R_{0}}\right)^{2 n}\right\}_{g_{n}^{R o}}^{R}=\left(\frac{R_{i}}{R_{0}}\right)^{n+1} s_{n}^{+}-\left(\frac{R_{i}}{R_{0}}\right)^{n+1} s_{n}-2 F_{n}^{R o}-2\left(\frac{R_{i}}{R_{0}}\right)^{n+1} F_{n}^{R i}
\end{aligned}
$$

The nonlinearities of the iron cause a change in the dipole field and the generation of higher harmonics. Both effects are expressed by the $\delta A_{n}$ for which we quote the final solution

- for $r<R_{i}$

$$
\begin{aligned}
& \delta A_{n}=\frac{1}{2 n}\left\{\left(\frac{r}{R_{i}}\right)^{n} g_{n}^{R i} R_{i}+\left(\frac{r}{R_{0}}\right)^{n} g_{n}^{R o} R_{0}+\left(\frac{r}{R_{i}}\right)^{n} s_{n}^{+} R_{i}\right\} \\
& \frac{d\left(\delta A_{n}\right)}{d r}=\frac{1}{2}\left\{\left(\frac{r}{R_{i}}\right)^{n-1} g_{n}^{R i}+\left(\frac{r}{R_{0}}\right)^{n-1} g_{n}^{R o}+\left(\frac{r}{R_{i}}\right)^{n-1} s_{n}^{+}\right\},
\end{aligned}
$$

- and for $r>R_{0}$

$$
\begin{aligned}
& \delta A_{n}=\frac{1}{2 n}\left\{\left(\frac{R_{1}}{r}\right)^{n} g_{n}^{R i} R_{i}+\left(\frac{R_{0}}{r}\right)^{n} g_{n}^{R_{0}} R_{0}+\left(\frac{R_{1}}{r}\right)^{n} s_{n} R_{i}\right\} \\
& \frac{d\left(\delta A_{n}\right)}{d r}=-\frac{1}{2}\left\{\left(\frac{R_{1}}{r}\right)^{n+1} g_{n}^{R i}+\left(\frac{R_{0}}{r}\right)^{n+1} g_{n}^{R_{0}}+\left(\frac{R_{1}}{r}\right)^{n+1} s_{n}\right\}
\end{aligned}
$$

If the accuracy of the solution obtained should be considered inadequate, it could be improved without great difficulty by a subsequent iteration procedure. 